\title{
Numerical and Experimental Investigations on the Aerodynamic Characteristic of Three Typical Passenger Vehicles
}

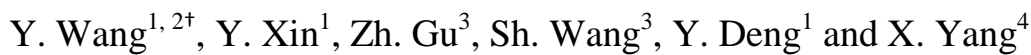 \\ ${ }^{1}$ Hubei Key Laboratory of Advanced Technology of Automotive Parts, Wuhan University of Technology, \\ Wuhan 430070, China \\ ${ }^{2}$ State Key Laboratory of Automotive Simulation and Control, Changchun 130025, China \\ ${ }^{3}$ State Key Laboratory of Advanced Design and Manufacturing for Vehicle Body, Changsha 430082, China \\ ${ }^{4}$ Wuhan Ordnance Noncommissioned officers School, Wuhan 430075, China \\ $†$ Corresponding Author Email: wangyiping@whut.edu.cn
}

(Received September 6, 2013; accepted November 11, 2013)

\begin{abstract}
The numerical simulation and wind tunnel experiment were employed to investigate the aerodynamic characteristics of three typical rear shapes: fastback, notchback and squareback. The object was to investigate the sensibility of aerodynamic characteristic to the rear shape, and provide more comprehensive experimental data as a reference to validate the numerical simulation. In the wind tunnel experiments, the aerodynamic six components of the three models with the yaw angles range from $-15^{\circ}$ and $15^{\circ}$ were measured. The realizable $k-\varepsilon$ model was employed to compute the aerodynamic drag, lift and surface pressure distribution at a zero yaw angle. In order to improve the calculation efficiency and accuracy, a hybrid Tetrahedron-HexahedronPentahedral-Prism mesh strategy was used to discretize the computational domain. The computational results showed a good agreement with the experimental data and the results revealed that different rear shapes would induce very different aerodynamic characteristic, and it was difficult to determine the best shape. For example, the fastback would obtain very low aerodynamic drag, but it would induce positive lift which was not conducive to stability at high speed, and it also would induce bad crosswind stability. In order to reveal the internal connection between the aerodynamic drag and wake vortices, the turbulent kinetic, recirculation length, position of vortex core and velocity profile in the wake were investigated by numerical simulation and PIV experiment.
\end{abstract}

Keywords: Vehicle aerodynamic, Wind tunnel experiment, Numerical simulation, PIV

\section{INTRODUCTION}

It is undoubted that the improvement of fuel efficiency in ground vehicles is currently, and will continue to be, a significant issue in the auto industry. At present, there are mainly two approaches to improve the fuel economy, one is to improve the combustion process in the engine (Abd-Alla 2002), and the other is to reduce the total drag force on the vehicle in motion (Jacques and Richard 2010, Frederique et al. 2004). In considering the latter, although the total drag force mainly consists of rolling resistance and aerodynamic drag, with a medium-size car, aerodynamic drag accounts for nearly 80 percent of the total drag force at $100 \mathrm{~km} / \mathrm{h}$. Moreover, the aerodynamic force is proportional to the square of the velocity, and the engine power required to overcome the aerodynamic drag is a function of the cube of the velocity. At high speeds, overcoming aerodynamic drag is responsible for more than 50 percent of fuel consumption (McCallen et al. 1999). There is therefore much scope for improving economy by reducing aerodynamic drag. However, in considering the aerodynamic drag force, a thorough analysis of the airflow around the vehicle, is a prerequisite. After which, a shape optimization methodology can be utilized to reduce the aerodynamic drag.

The aerodynamic drag in ground vehicle includes form drag, skin friction, interference drag, induced drag and cooling drag. The form drag due to the flow separation around the vehicle body contributes to 50 to 65 percent to the overall aerodynamic drag. The flow around vehicles is highly threedimensional, dominated by large separation regions, large and small vortices, and complex recirculation regions. The main contributions to aerodynamic drag in a bluff body type vehicle arise from $\mathrm{s}$ e $\mathrm{p}$ a $\mathrm{r}$ a $\mathrm{t}$ e $\mathrm{d}$ f 1 o 
the rear causing pressure recovery losses and the creation of vorticity in the wake (Khalighi et al. 2001), and the contribution due to the rear part is often over 80\%( Kourta and Gilieron 2009). The structure of the wake is dependent on the geometry of the vehicle upstream of the blunt base edge (Howell 1975, Morel 1978, Ahmed 1983). There are essentially three different vehicle configurations: squareback, fastback and notchback. These geometries differ primarily in the angle of the slanted rear window with the squareback essentially a fastback with a zero angle of base slant and the notchback a fastback with a boot lid.

In response to the first fuel crisis of the 1970s, a new focus on aerodynamics was instigated across the automobile industry as a part of a strategy to reduce fuel consumption, and part of this focus was an investigation into the fundamental flow characteristics of automobile bluff bodies. Likewise, it was realized that the aerodynamic drag of road vehicles was dominated by the pressure drag due to the flow separation at the rear end of the body (Morel 1978), and there had been concern about the influence of the rear configuration on aerodynamic characteristics (Hucho 1993, Song et al. 2012). Generally, in the wake of road vehicle, there exist a recirculation region which is characterized by two small inner vortices and a pair of streamwise longitudinal vortices (Ahmed 1983). For a squareback which is typical for SUV or miniVan, a region recirculation is the dominant feature because there are no slanted edges around which a longitudinal vortex may form (Richards 2002). Moreover, because of the large separation region in the wake region, the contribution to the aerodynamic drag will be more. For fastback geometry, the works of Janssen and Hucho (1975), Morel (1978) and Ahmed (1984) were amongst the first to investigate the relation between the shape of a vehicles rear end and the aerodynamic drag, and identify the significance of rear slanted angle on aerodynamic characteristics. Their results showed that the wake topology and aerodynamic drag was strongly depended on the angle of the slanted rear window $\alpha$, and there was a critical angle $\left(\alpha \approx 30^{\circ}\right)$. For angles less than the critical angle, the flow first separated from the roof of the body, then reattached on the slanted surface, and then separated again at the rear end of the model. As the angles increased and approached $30^{\circ}$, the form drag of the vehicle increases sharply. For larger angles the drag drops again, and it remains almost constant with further increased in $\alpha$. Compared to the other two rear end geometries, for a notchback vehicle which is more typical for passenger car, the complex wake structure behind the notchback is by far the least well understood. It was found that the drag coefficient changed with rear geometry was less extreme for notchbacks than for fastbacks (Hoffman et al. 2001). The notchback vehicles exhibit a complicated near-wake flow, and the structure of which is still not understood (Gilhome et al. 2001). The airflow around a notchback vehicle can be characterized by two different types of flow separation: quasi-two-dimensional and threedimensional (Hucho 1998). Jenkins (2000) concluded from the wind tunnel testing that there existed two streamwise vortices that extend from the deck lid surface toward the center and downstream. Gilhome (2001) proposed a new topological structure for the wake that the wake consisted of large hairpin and shear-layer vortices which were regularly shed, a stable re-circulation vortex and the well-know C-pillar vortices. Gaylard et al. (2007) presented a series of observations of time-averaged wake asymmetry for some notchback vehicle geometries. The mechanism that induces the asymmetry needs to be further researched; though it has been theorized that it may be related to the rear end shape. In order to explore the internal connection between the wake and aerodynamic characteristic, Sims-Williams et al. (2001) investigated the links between notchback geometry, aerodynamic drag, flow asymmetry and unsteady wake structure systematically. Without exception, most of the previous studies were focused on the aerodynamic drag while the other aerodynamic forces were rarely mentioned. Actually, the other aerodynamic forces are closed to the overall vehicle performance, such as the side force to the crosswind stability and so on. Therefore, in current research, the sensibility of the other aerodynamic force to the rear shape will be investigated and more comprehensive experimental data will be provide as reference to investigate the change law between the different rear shape and aerodynamic characteristic.

A lot of attention had been paid to the wake characteristic of squareback, fastback and notchback, but these researches were based on individual models. Because the overall configurations were difference, it was very difficult to verify the influence of rear configuration. In order to investigate the influence of the rear configuration on aerodynamic characteristics systematic, and compare the wake topologies of different rear configuration intuitively. In current research, a group of models which named MIRA reference cars (Le Good and Garry 2004), which had the same configuration in front but the rear parts were squareback, fastback and notchback, were employed. The numerical simulation combined with wind tunnel test was employed to investigate the aerodynamic characteristics and the wake structure.

In the past two decades, Computational Fluid Dynamics (CFD) has been used widely in vehicle aerodynamic studies (Himeno and Fujitani 1993, Jindal et al. 2005, Murad et al. 2004 and Basara et al. 2012). Over this period major advances in CFD codes, computational algorithms, physical models and methods, high performance computing algorithms and supporting computer hardware had led to a widespread acceptance of CFD as a viable tool for aerodynamic development. It was generally accepted that the CFD tools provide sufficient accuracy to support aerodynamic development. As a result, nearly every automotive manufacture today made significant use of CFD for design and optimization of vehicle shapes. The CFD also showed a unique advantage to visualize the structure of steady and unsteady wakes, and could 
provide more information which was very difficulty to obtain in the wind tunnel test. This information would be very helpful to understand the link between the aerodynamic characteristic and the flow field.

\section{MIRA REFERENCE CAR}

The MIRA reference cars were a group simplified car shapes which were evolved from work undertaken in the early 1980s when European and North American wind tunnel operators began a series of correlation exercises (Le Good and Garry 2004). Initially the model was constructed in $20 \%$, $25 \%, 1 / 3 \mathrm{rd}$ and full-scale versions. In 1990s, 40\% and $30 \%$ versions were added to MIRA's own collection to aid manufacturer studies in model-full to full-scale correlation. With the availability of published experimental data and the advantage of simple surface geometry, the MIRA reference car became a popular test case when CFD emerged as a tool for automobile aerodynamics. In current researches, MIRA reference cars with 1:3 scales were employed in the wind tunnel tests and numerical simulation. The related aerodynamic parameters and wake flow were measured in the HD-2 wind tunnel which will be introduced in the Experimental Setup section to validate the numerical scheme, and the Fig. 1 shows the three views of the three available variants, namely, fastback, notchback and squareback.

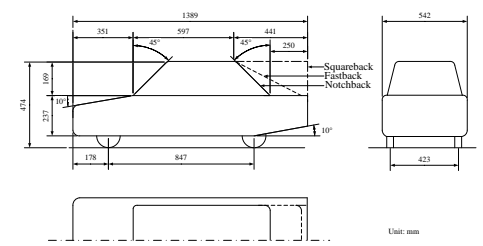

Fig. 1. The three views of the three available variants, namely, fastback, squareback and notchback

\section{EXPERIMENTAL SETUP}

The experiments were carried out in HD-2 Boundary Layer Wind Tunnel (HD-2BLWT) in Wind Engineering Research Center, Hunan University. The wind tunnel is a horizontal closedcircuit type wind tunnel which is configured with two closed test sections. The high speed section has a cross-sectional area of $3 \times 2.5 \mathrm{~m}^{2}$, and the maximum wind velocity in the test section is $58 \mathrm{~m} / \mathrm{s}$ provided by its $617 \mathrm{kw}$ propeller. The other is the low speed section with the cross-sectional area of $5.5 \times 4.4 \mathrm{~m}^{2}$, and the maximum wind velocity is $18 \mathrm{~m} / \mathrm{s}$. The current tests were performed in the high speed section. In order to eliminate the ground boundary layer, a boundary layer pumping system was installed in front of the test model. The frontal area of the MIRA model resulted in a blockage ration of about $2.75 \%$ which meets the requirement that the blockage ratio of the experimental model should be less than 5\% (Lee and Choi 2000). The average turbulence intensity is less than $0.2 \%$. In current research, a floating-frame strain gauge sixcomponent balance (Fig.2) is employed to measure the aerodynamic force. In order to ensure the accuracy of the experimental results, the balance is calibrated in ground coordinate system by the manufacturer semiannually. Moreover, before installing the measurement model, a five kilogram weight was loaded on the balance to verify the accuracy of the results, and the whole system was returned zero before sampling. The test vehicle models were installed on a six-component balance (Fig. 3).

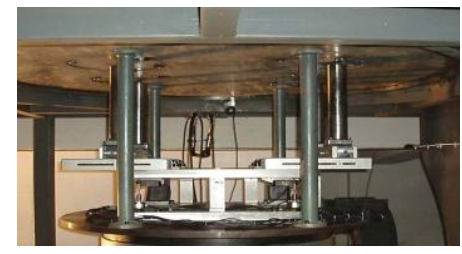

Fig. 2. Floating-frame strain gauge sixcomponent balance

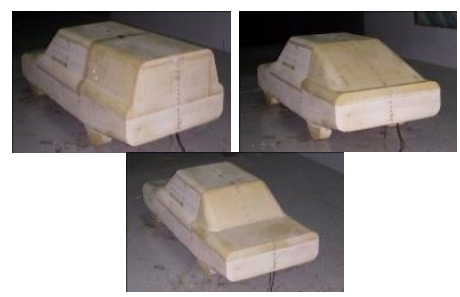

Fig. 3. The test models located on the sixcomponent balance

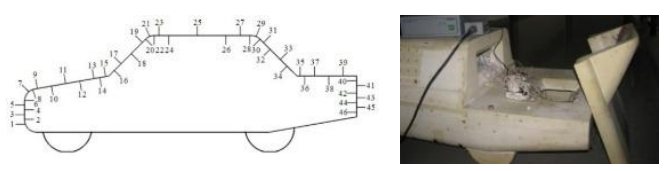

Fig. 4. The locations of sensors along the symmetry plane and interior connection

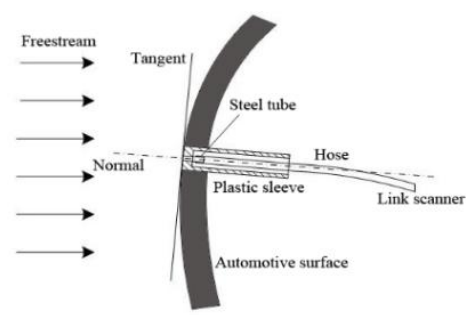

Fig. 5. The schematic diagram of the pressure tap

The data obtained in current experiment included the aerodynamic force and moment, surface pressure, as well as mean velocities in the wake. In order to measure the pressure distribution along the symmetry plane, about 46 pressure taps were placed on the symmetry plane (Fig.4). The pressure taps are1-mm-diam steel tubes which are flushed with the automotive surfaces to ensure the smoothness, and the steel tube is linked to the DTCnet electronic pressure scanner with hoses. In order to ensure the steel tube is tight, a plastic sleeve is covered over the steel tube (Fig.5). The data were first stored in the computer memory, and then transferred into the hard disk. The measured mean pressures are used to determine the pressure coefficient defines as (Hucho 1998), 


$$
C_{P}=\frac{P-P_{r e f}}{\frac{1}{2} \rho v_{\infty}^{2}}
$$

Where $P_{r e f}$ is the reference pressure, $P$ is the measured mean pressure. In the current experiment, the reference pressure is the static pressure measured at the fillets. In order to investigate the Reynolds number effect, the corresponding experimental data were collected with free stream velocities at $15 \mathrm{~m} / \mathrm{s}, 20 \mathrm{~m} / \mathrm{s}, 25 \mathrm{~m} / \mathrm{s} 30 \mathrm{~m} / \mathrm{s}, 40 \mathrm{~m} / \mathrm{s}$ respectively.

The measurements for the velocity fields in the near wake of the model were carried out by a non-timeresolved 2D-1C system. The system was distributed by Beijing Li Fang Tian Di (BLFTD)Technology Development Ltd. and consisted of a double NdYag laser from Beamtech that produced laser pulses $(532 \mathrm{~nm}$, peak energy of $500 \mathrm{~mJ} /$ pulse,) $6-8 n \mathrm{~s}$ duration with a repeatability frequency of $10 \mathrm{~Hz}$ ) to illuminates the measurement position by forming a 0.5 -mm-thick $\times 600 \mathrm{~mm}(y) \times 400 \mathrm{~mm}(x)$ laser sheet (Fig.6). A CCD camera (IPX-11M-GC camera) of $4000 \times 2672$ pixels resolution records particle images, operated under double exposure mode at a sampling rate of $5 \mathrm{~Hz}$, and the spatial resolution of the camera was $9 \mu \mathrm{m} \times 9 \mu \mathrm{m}$. The synchronization was solved by a compact system MicroPulse 725 provided by Vision Asia. The time delay between the two pulses is set to $35 \mu \mathrm{s}$ for the free stream velocity at $30 \mathrm{~m} / \mathrm{s}$. The flow was seeded with $1 \mu \mathrm{m}$ droplets of di-ethyl-hexyl-sebacate (DEHS) which was atomized by the compressed air feed. The seeding was injected into the airflow in a single pass by means of a smoke rake positioned upstream of the nozzle contraction where it provided minimal disruption to the air flow upstream of the model. The optical setup was placed at the top of the wind tunnel and the CCD camera was placed outside the test section. The measurement position was located at the longitudinal symmetry plane and the nearwake and the far-wake, a distance of approximately $1200 \mathrm{~mm}$ in the streamwise direction, was divided into four different fields-of-view with a $75 \mathrm{~mm}$ overlap between the images. Two hundred doubleframe images were typically acquired for each segment of the wake. The MicroVec V2.3, developed by BLFTD, was used to acquire and analyze the PIV image data. An autocorrelation method was used with an interrogation spot size of 64 by 64 pixels and 50\% overlap, and the grid spacing of the PIV measurements is 32 pixels

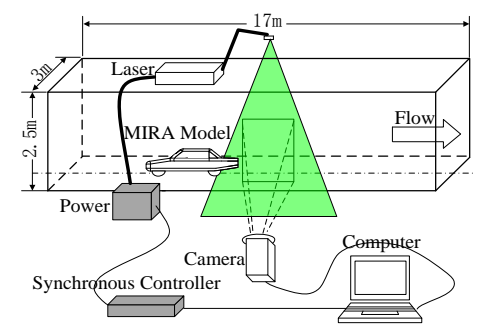

Fig. 6. The schematic diagram of the PIV setup for velocity measurement in symmetry plane

\section{NUMERICAL METHOD}

The road vehicles are low Mach number transport tools, and the present research mainly was carried out to investigate the steady aerodynamic characteristic of these vehicles. So the steady state Reynolds Averaged Navier-stokes (RANS) was employed in current research. Compared to other turbulence models, the realizable $k-\varepsilon$ (Shin et al. 1995) model was more accurate to predict the related aerodynamic parameters in vehicle aerodynamics (Wojciak et al. 2012). In this model, a new model dissipation rate equation (based on the mean-square vorticity fluctuation at large turbulent Reynolds number) and a new realizable eddy viscosity formulation were constructed. Therefore, this model is more suitable for a variety of flows, including mixing layers, planar and round jets, rotating homogenous shear flows, boundary layers with adverse pressure gradients and flows with separation induced by the geometry of the domain. For a steady incompressible flow, the modeled transport equation for $k$ and $\varepsilon$ were given by (Shin et al. 1995):

$$
\begin{aligned}
& \frac{\partial\left(u_{i} \varepsilon\right)}{\partial x_{i}}=\frac{\partial}{\partial x_{i}}\left[\left(v+\frac{v_{t}}{\sigma_{\varepsilon}}\right) \frac{\partial \varepsilon}{\partial x_{i}}\right]+C_{1} S \varepsilon-C_{2} \frac{\varepsilon^{2}}{k+\sqrt{v \varepsilon}} \\
& \frac{\partial\left(u_{i} k\right)}{\partial x_{i}}=\frac{\partial}{\partial x_{i}}\left[\left(v+\frac{v_{t}}{\sigma_{k}}\right) \frac{\partial k}{\partial x_{i}}\right]+P_{k}-\varepsilon \\
& v_{t}=C_{\mu}^{*} \frac{k^{2}}{\varepsilon} \\
& C_{\mu}^{*}=\frac{1}{A_{0}+A_{s} U} \frac{(2)}{\varepsilon} \\
& C_{1}=\max \left[0.43, \frac{\eta}{\eta+5}\right] \eta=S \frac{k}{\varepsilon}, U^{*} \equiv \sqrt{S_{i j} S_{i j}+\tilde{\Omega}_{i j} \tilde{\Omega}_{i j}}, \\
& \tilde{\Omega}_{i j}=\frac{1}{2}\left(\frac{\partial u_{i}}{\partial x_{j}}-\frac{\partial u_{j}}{\partial x_{i}}\right), A_{s}=\sqrt{6} \cos \phi, \phi=\frac{1}{3} \cos ^{-1}(\sqrt{6} W) \\
& W=\frac{S_{i j} S_{j k} S_{k i}}{\tilde{S}^{3}}, \quad \tilde{S}=\sqrt{S_{i j} S_{i j}}, \quad S_{i j}=\frac{1}{2}\left(\frac{\partial u_{j}}{\partial x_{i}}+\frac{\partial u_{i}}{\partial x_{j}}\right) \\
& \sigma_{k} \equiv 1.0, \sigma_{\varepsilon} \equiv 1.2, C_{2} \equiv 1.9, A_{0} \equiv 4.0
\end{aligned}
$$

where $k$ is the turbulence kinetic energy, $\varepsilon$ is the dissipation rate of turbulence energy, $P_{k}$ is the shear production of turbulent kinetic energy, $v_{t}$ is the turbulent eddy viscosity, $S$ is the modulus of the mean rate-of-strain tensor, $v$ is the kinetic viscosity, $u_{i}(i=1,2,3)$ is the velocity component, $x_{i}(i=1,2,3)$ is cartesian coordinates

The computational domain and coordinate system for the simulations is shown in Fig.7. The computational domain was a cuboid and had the same size as the high speed test section of the HD-2 wind tunnel (Fig.6), and the location of the model in the domain was also the same. The coordinate was the same as the experiment, and the origin was located at $0.4750 \mathrm{~m}$ below the ground in y direction, at the middle of vehicle length in $\mathrm{x}$ direction and at the middle of wheelspan in $\mathrm{z}$ direction. In current research, the numerical simulation was an effective complement to the wind tunnel experiment. 
Therefore, in order to reflect experimental conditions, the boundary conditions imposed to the domain were as follow: 1) a constant velocity of $U_{0}$ at boundary one, 2) pressure outlet with a zero gauge pressure at boundary four, 3) a no slip wall was imposed to the boundary two, boundary five and the side wall of the cuboid domain, 4) in the experiment, the ground boundary layer was eliminated by the pumping system, in order to eliminate the ground boundary layer in the simulation. The boundary three was treated with a moving wall at the same speed $U_{0}$. The solution algorithm for the simulation was based on the well known SIMPLE algorithm for the iterative solution of the steady RANS equations, and the algorithm was of second-order upwind scheme in spatial discretization. All the simulations were fulfilled by the commercial software package Ansys Fluent 14.0.

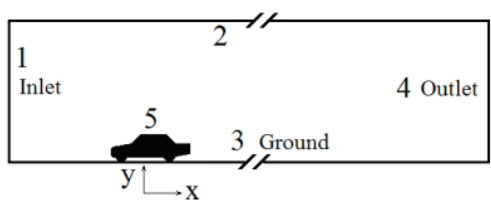

Fig. 7. The computational domain

Generally speaking, structured meshes offer simplicity and easy data access, while unstructured meshes offer more convenient mesh adaptively and a better fit to complicated domains. High-quality hybrid meshes enjoy the advantages of both approaches (Marshall and Paul 2000). In current computations, the hybrid Tetrahedron-HexahedronPentahedral prism mesh was employed to compute the flow field around the vehicles, and the mesh was generated by Ansys ICEM CFD 14.0. In the computational domain, a small cuboid was constructed to surround the vehicle model. The tetrahedral mesh was generated inside the small cuboid, and the hexahedral mesh was generated outside the small cuboid. The hexahedral and tetrahedral elements were joined by inserting pyramidal elements in the interface. Three layers prism elements were generated near the vehicle surface to provide an accurate estimation of the velocity profile near the wall, when using the wall function, by keeping the $\mathrm{y}^{+}$value within an acceptable range (20-200) (Connor et al. 2006). The numerical grid in the symmetry plane in the vicinity of the body is shown in Fig. 8. It consisted of about five million cells, and local grid refinement was applied near the body surface and in the wake region. Further grid refinement showed little difference in the results reported here.

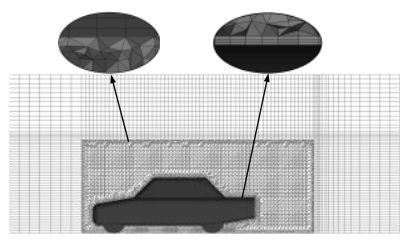

Fig. 8. Numerical grid in the symmetry plane in the vicinity of the body

\section{RESULTS AND DISCUSSION}

\subsection{Aerodynamic Force}

In vehicle aerodynamic, the aerodynamic characteristic is reflected by the aerodynamic force coefficient. In current research, the aerodynamic coefficients were obtained by experiment and numerical simulation to compare the aerodynamic characteristic of the different rear shape. The dimensionless aerodynamic force coefficients were defined as

Drag coefficient: $C_{D}=F_{x} / \frac{1}{2} \rho v_{\infty}^{2} \cdot S$

Lift coefficient: $C_{L}=F_{y} / \frac{1}{2} \rho v_{\infty}^{2} \cdot S$

Lateral force coefficient: $C_{Z}=F_{Z} / \frac{1}{2} \rho v_{\infty}^{2} \cdot S$

Rolling moment coefficient:

$C_{M X}=M_{X} / \frac{1}{2} \rho v_{\infty}^{2} \cdot S \cdot W B$

Yawing moment coefficient:

$C_{M Y}=M_{y} / \frac{1}{2} \rho v_{\infty}^{2} \cdot S \cdot W B$

Pitching moment coefficient:

$C_{M Z}=M_{z} / \frac{1}{2} \rho v_{\infty}^{2} \cdot S \cdot W B$

Where $F_{x}, F_{y}, F_{z}$ are the aerodynamic drag force, lift force and lateral force respectively, $M_{x}, M_{y}, M_{z}$ are the rolling moment, yawing moment and pitching moment respectively, $\rho$ is the air density $\left(1.2471 \mathrm{~kg} / \mathrm{m}^{3}\right.$ in present study), $S$ is the frontal area $\left(0.2064 \mathrm{~m}^{2}\right.$ in present study), $v_{\infty}$ is the incoming flow velocity, $W B$ is the wheel base $(0.8470 \mathrm{~m}$ in present study).

Table.1 shows the drag coefficient and lift coefficient obtained by numerical simulation and experiment, and the results between the wind tunnel test and simulation shows a good agreement. Generally, the order of magnitude of the Reynolds number

$R e_{W B}=\frac{\rho \cdot v_{\infty} \cdot W B}{\mu}\left(\mu=1.7894 \times 10^{-5}\right)$

in the full-scale vehicle test would approach to $10^{6}$ (Wiedemann and Ewaldt 1989), and the dependence of the drag coefficients on Reynolds number was very small and sudden changes do not occur (Hucho 1998). Therefore, in wind tunnel measurements and simulation, in order to ensure the reliability of the results, the Reynolds number should be approach to the same order of magnitude $10^{6}$ and be larger than some a critical Reynolds number. In current experiments the drag variation is quite small when the wind velocity over $25 \mathrm{~m} / \mathrm{s}$, which corresponding to the Reynolds number $1.4758 \times 10^{6}$.

It is well know that the Mira model group is often introduced to validate the reliability of the experimental and computational results. In order to deal with the corrections for automotive model tests in the TJ-2 wind tunnel of Tongji University, Pang et al. (2002) ever measured the drag coefficient in TJ-2 and IVK automotive model wind tunnel respectively. Hoffman et al. (2001) employed the Mira model group to investigate the effect of test section configuration on aerodynamic drag 
measurements. Therefore, the experimental results obtained by Pang et al.(2002) and Hoffman et al. (2001) were introduced to validate the reliability of HD-2 and computation. The results revealed that the current results were acceptable in spite of some difference which may be induced by the different blockage ratio, Reynolds number and ground effect in different wind tunnel.

Table 1 The aerodynamic force coefficient of models.

\begin{tabular}{|c|c|c|c|c|c|c|c|}
\hline & \multicolumn{4}{|c|}{$C_{D}$} & \multicolumn{2}{c|}{$C_{L}$} \\
\hline & HD-2 & TJ-2 & IVK & Hoffman & CFD & HD-2 & CFD \\
\hline Fastback & 0.2849 & 0.2631 & 0.2795 & $\approx 0.26$ & 0.2738 & 0.0460 & 0.0410 \\
\hline Notchback & 0.3183 & 0.3016 & 0.3204 & $\approx 0.29$ & 0.3048 & 0.0416 & 0.0397 \\
\hline Squareback & 0.3842 & 0.3668 & 0.3874 & $\approx 0.36$ & 0.3742 & -0.3633 & -0.3592 \\
\hline
\end{tabular}

The aerodynamic parameters of the three typical vehicles showed that the drag coefficient of the squareback was the largest, and the fastback was the smallest. Therefore, in the viewpoint of fuel economy, the fastback is the best choice. For the lift coefficient, the notchback and fastback were positive, and the squareback was the negative. It is well know that, for high speed vehicles, too much lift force will decrease the adhesion force of tires, and make the car out of control. Therefore, in many vehicle designs especially for Formula One, in order to guarantee the excellent high speed operational stability and dynamics, the negative lift force was often sought. So in the viewpoint of high speed operational stability, the squareback is the most suitable selection, but how to reduce the aerodynamic drag coefficient also became the key for its application.

According to the theory of vehicle aerodynamic, the form drag was the major source of aerodynamic drag, therefore, the investigation related to the surface pressure distribution was significant to reveal the mechanism of form drag, specify the optimum position of air intake and check the rationality of shape design. Fig.9 shows the pressure distribution along the upper center-line of the three typical vehicles, and the results between CFD simulation and wind tunnel measurement showed a good agreement. Because of the same front shape, the pressure distribution was almost the same and varied as expected. It started from the stagnation point, followed by a rapid drop in pressure due to the transition at the edge of the hood, until it reached the intersection of the hood and the windshield where the pressure reaches its second peak. The pressure then relaxed over the windshield, and reached the second low point at the transition between the windshield and the cabin top. The pressure recovered over the cabin top of the model, for the notchback and fastback, the pressure reached the third low point at the transition between the cabin top and the rear window. Over the rear window and luggage compartment, pressure recovered. Because of the radical differences in the rear shapes, there was a large difference in pressure distribution. In order to describe the pressure distribution more visually, the pressure coefficients was depicted on the vehicle surface (Fig.10). The schematic diagram obviously revealed that the area of rear negative pressure zone of the squareback model was the largest and the fastback model was the smallest. Therefore, the drag coefficient of the squareback was the largest, and the fastback was the smallest

For the current three models, their bottoms were the same, so the pressure distributions along the lower centerline were also the same (Fig.9). Therefore, the lift force difference among the models was mainly induced by the rear shape. According to the definition of the lift force, the distance between the upper centerline and lower centerline could qualitatively reflect the lift force level. It would be persuasive to conclude that the less the sum distance was, the less the lift force was, and when the sum distance is minus, the lift force could be negative. For the squareback, there was reason to believe that the sum distance between the upper centerline and lower centerline was minus.

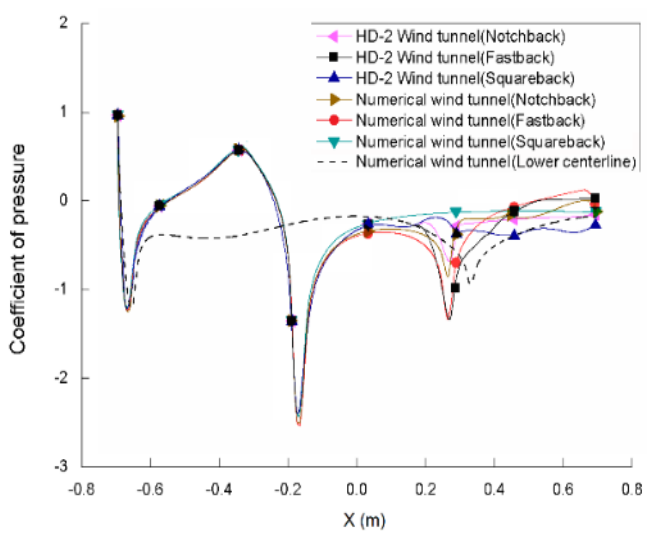

Fig. 9. The pressure distribution along the upper centerline.

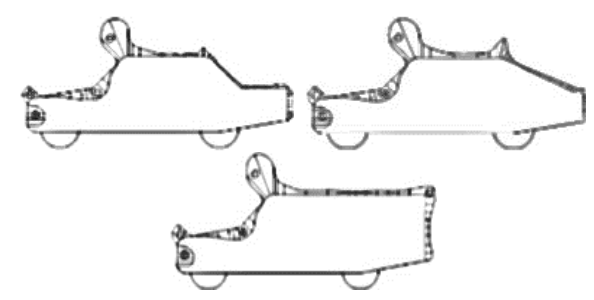

Fig. 10. The schematic diagram of the pressure coefficient along the upper center-line ("+" means positive and "." means negative). 
.In the actual situations, the wind direction was not always parallel with the driving direction, and the yaw angle was often existent. Therefore, in present study, the variation of the six aerodynamic force coefficients with the yaw angle was investigated by the wind tunnel experiment. The yaw angle $(\beta)$ varied from $-15^{\circ}$ to $15^{\circ}$, and the wind speed was $30 \mathrm{~m} / \mathrm{s}$.

Figure 11 shows the relationship between the aerodynamic drag coefficient $\left(C_{D}\right)$ and yaw angle $(\beta)$. The result revealed that the $C_{D}$ was increased with the increase of $|\beta|$, and when the $|\beta|$ exceeded $9^{\circ}$, the increment of the $C_{D}$ decreased gradually. Moreover, the results also revealed that the drag coefficient of the fastback was the least sensitive to the change of the yaw angle, while the notchback was the most sensitive. In theory, the $C_{D^{-}} \beta$ curve should be symmetrical when the yaw angle ranged from $-15^{\circ}$ to $15^{\circ}$, but in the actual wind tunnel experiment, the flow field of wind tunnel and the vehicle model were not always symmetrical. Therefore, the results obtained by the experiment were not symmetrical strictly.

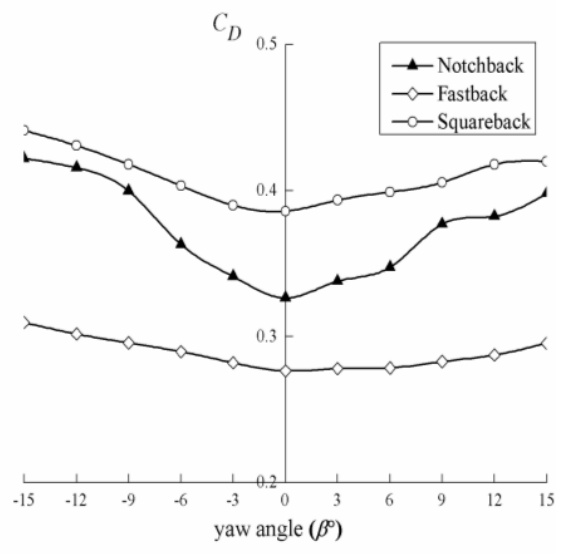

Fig. 11. The $C_{D^{-}} \beta$ relationship curve

Figure 12 shows the relationship between the aerodynamic lift coefficient $\left(C_{L}\right)$ and yaw angle $(\beta)$. The results revealed that, with the change of the yaw angle, the lift coefficients of the notchback, fastback and squareback had the similar change rule. When $|\beta|$ varied from $0^{\circ}$ to $3^{\circ}$, the $C_{L}$ decreased, but when $|\beta|$ varied from $3^{\circ}$ to $15^{\circ}$, the $C_{L}$ increased gradually and the increment become bigger and bigger. Moreover, the results also revealed that the shape of the vehicle was more streamlined, and the lift coefficient was higher.

Figure 13 shows the relationship between the aerodynamic lateral force coefficient $\left(C_{Z}\right)$ and yaw angle $(\beta)$. The results revealed that the relationship between the $C_{Z}$ and $\beta$ was almost linear. The lateral force was related with the side projection area, so in the same yaw angle, the lateral force of the squareback was the biggest, and the fastback was the smallest.

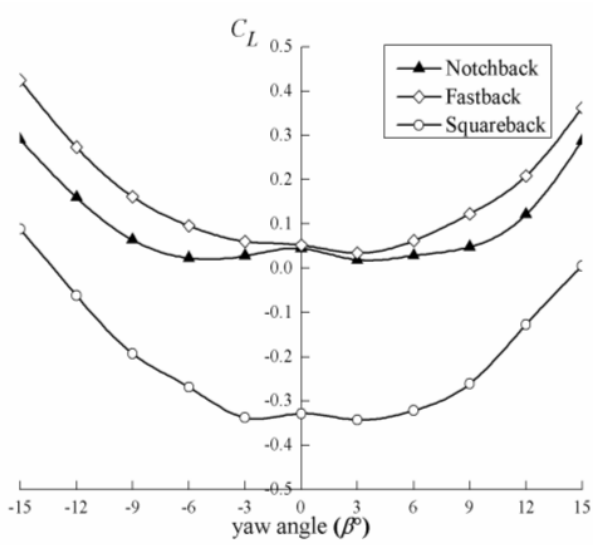

Fig. 12. The $C_{L^{-}} \beta$ relationship curve

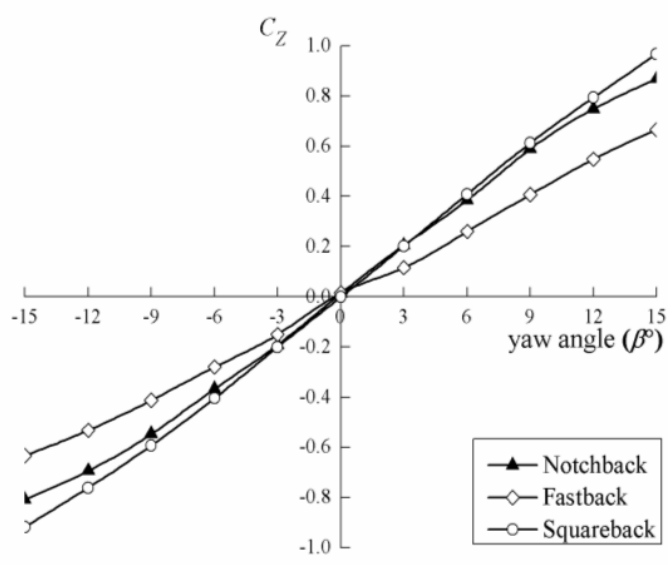

Fig. 13. The $C_{Z^{-}} \beta$ relationship curve

Figure 14 shows the relationship between the aerodynamic yawing moment coefficient $\left(C_{M Y}\right)$ and yaw angle $(\beta)$. The results revealed that the $C_{M Y}$ was proportional to $\beta$, and in the same yaw angle, the yawing moment coefficient of the squareback was the smallest, while the fastback was the biggest. In other words, comparing with the fastback and notchback, the crosswind stability of squareback was better.

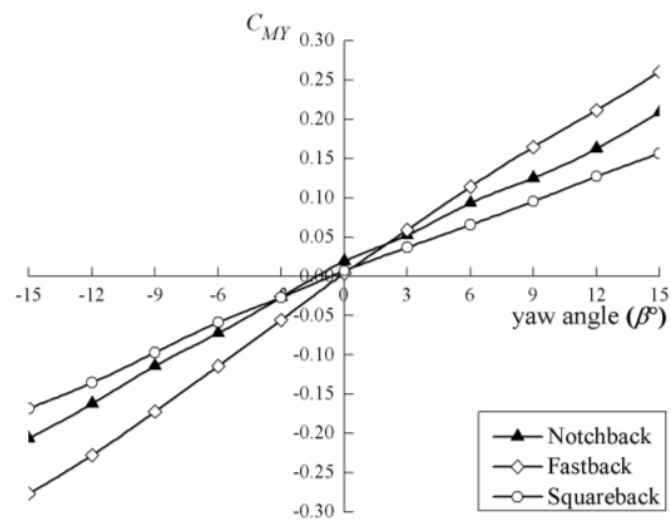

Fig. 14. The $C_{M Y^{-}} \beta$ relationship curve 
Figure 15 shows the relationship between the aerodynamic rolling moment coefficient $\left(C_{M X}\right)$ and yaw angle $(\beta)$. The results revealed that the $C_{M X}$ was proportional to the $\beta$, and in the same yaw angle, the rolling moment coefficient of the squareback was the biggest, and the fastback was the smallest. In other words, the more streamlined model would result in smaller change of the $C_{M X}$ over yaw angle. Theoretically, the $C_{M Y}, C_{M X}$ and $C_{Z}$ should be zero when the yaw angle approaches zero. However, because of the structural error of wind tunnel and the machining errors of the vehicle model, the flow field around the vehicle model is not always symmetrical. Therefore, in the actual wind tunnel experiment, the $C_{M Y}, C_{M X}$ and $C_{Z}$ often are not zero when the yaw angle approaches zero.

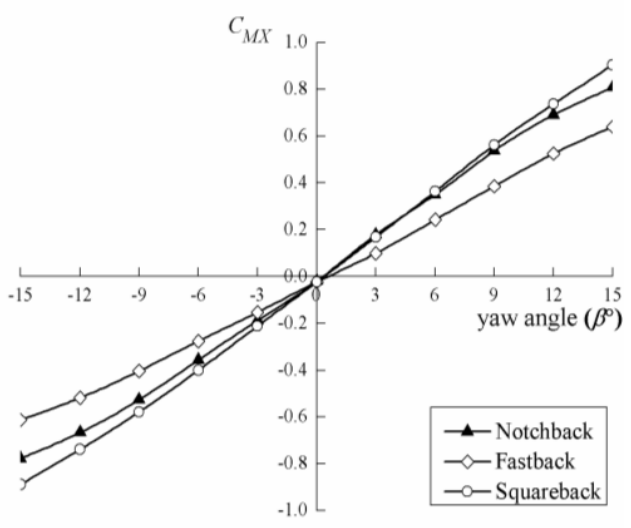

Fig. 15. The $C_{M X^{-}} \beta$ relationship curve

Figure 16 shows the relationship between the aerodynamic pitching moment coefficient $\left(C_{M Z}\right)$ and yaw angle $(\beta)$. The results revealed that the $C_{M Z}$ was minus, and $\mid C_{M Z}$ |was increased with the increase of $|\beta|$. The change of the notchback was the smallest, while the change of the squareback was the biggest.

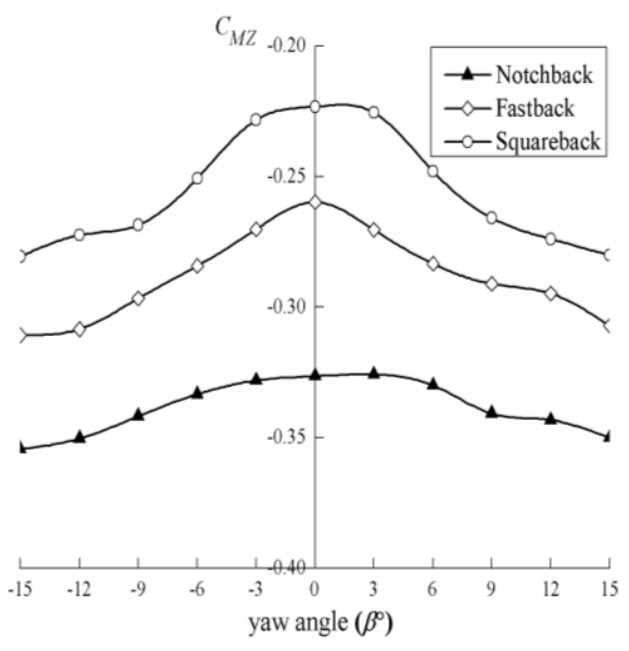

Fig. 16. The $C_{M Z}-\beta$ relationship curve

\subsection{Wake Structure}

It was well know that the rear-end shape of a car was one of the most important elements which governed the aerodynamic characteristics, and there were some experimental studies related to the wake structure of road vehicles (Al-Garni et al. 2008, Kim et al. 2008, Al-Garni et al. 2004). In current research, the wind tunnel experiment and CFD were employed to investigate the wake structure of the three typical passenger vehicles. Most wake studies for automotive applications involved the very near wake close to the reverse flow 'bubble', or 'deadwater' region, immediately behind the vehicle, and perhaps extending a further body length downstream. The region of interest here also followed this example.

Figure 17 shows the time-averaged velocity distribution along the longitudinal symmetry plane in a viewing window $1200 \mathrm{~mm}$ long and $520 \mathrm{~mm}$ high obtained by PIV and CFD, and averaging is done over two hundred images in PIV. The results revealed that the velocity distribution obtained by numerical simulation was similar to the experimental. In general, for a moving vehicle, there was a 'vacuum pocket' (the vacuum means the velocity approaches to the zero) in its wake. The shape of vehicle determined its tendency to build up frontal pressure and its coefficient of drag, which was how large of a 'vacuum pocket' it left in its wake. In current research, the three vehicles had the same front shape, so the aerodynamic differences were induced by the rear shape. For the fastback, the flow attached on the back and downwash along it, thus the size of the 'vacuum pocket' was quit small in the wake. For the squareback, the area of the eddy diffusion was quite large, so the size of the 'vacuum pocket' was quit large. For the notchback, an additional 'vacuum pocket' was produced by the flow recirculation behind the rear windshield.
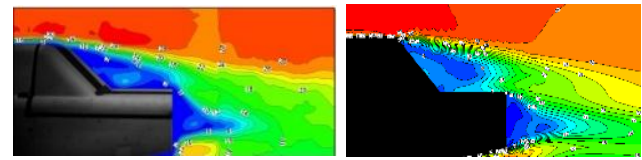

(a) Notchback
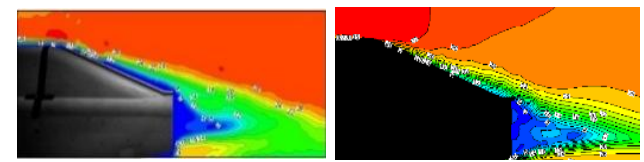

(b) Fastback
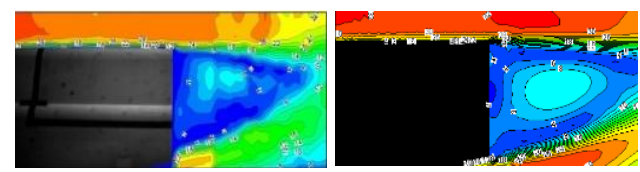

(c) Squareback

Fig. 17. Velocity distribution along the longitudinal symmetry plane (Left: HD-2, Right: CFD)

Figure 18 presents a direct comparison between measured and predicted recirculation region structure at the model centerline. Although the 
streamlines depicting the measured recirculation region structure was somewhat smaller than the prediction, the comparison nevertheless showed the numerical method made very similar and good prediction of the general two-tier recirculation region vortex structure, and the position of the vortex core.

By comparing the vortex structure of the three different backs, it was found that:

1) The structure and position of the wake vortex was different depending on the rear shape. Except for a clockwise vortex induced by the step in the notchback, the wake flow field of the model group could be composed of two parts that were the downwash shear flow from the back and the upwash shear flow from the bottom. For convenience, the upper clockwise vortex was defined as vortex A, and the lower anti-clockwise vortex was defined as vortex $\mathrm{B}$.

2) For the position of vortex core, the vortex $A$ of notchback was located at $(760 \mathrm{~mm}, 680 \mathrm{~mm})$, and the vortex B located at $(760 \mathrm{~mm}, 600 \mathrm{~mm})$. The fastback almost had the same vortex core position with the notchback. However, the vortex core of the squareback was dragged quite far from the body, and the vortex A located at $(1010 \mathrm{~mm}, 880 \mathrm{~mm})$, the vortex B located at $(910 \mathrm{~mm}, 680 \mathrm{~mm})$. Because the vortex core was far from the body, the range of the wake vortex was expanded and the drag coefficient also will increase. Therefore, it could be concluded that the greater distance between the vortex core and the car body, the lager the drag coefficient.

3 ) It could be found that the diffusion lengths of the fastback and notchback were almost the same except for the vortex behind the rear windshield in notchback, and the diffusion length was about $200 \mathrm{~mm}$, and the squareback was about $400 \mathrm{~mm}$. because of the large relative velocity between the vortex boundary and freestream, combined with the air viscosity, a turbulence boundary layer would be produced on the vortex boundary. In the turbulent boundary layer, the energy exchange could take place drastically, and the energy dissipation would decrease along with the decrease of the area of boundary layer. In the view of the energy, the area of the turbulence boundary layer of the fastback was the minimum, and the squareback was the maximum. Therefore, the drag coefficient of the fastback was the least, and the squareback was the largest.

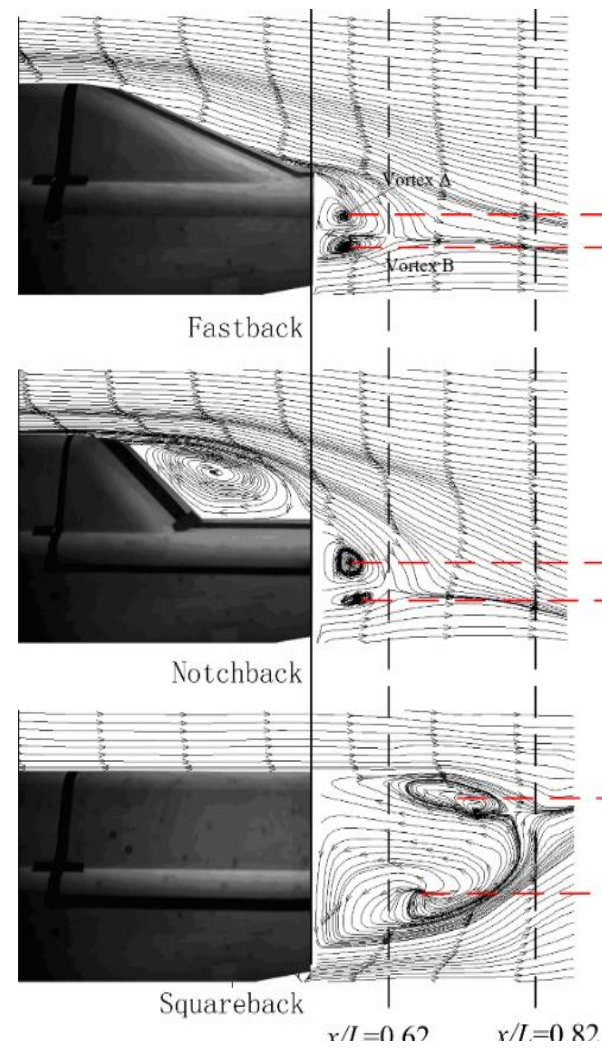

(a) HD-2 wind tunnel

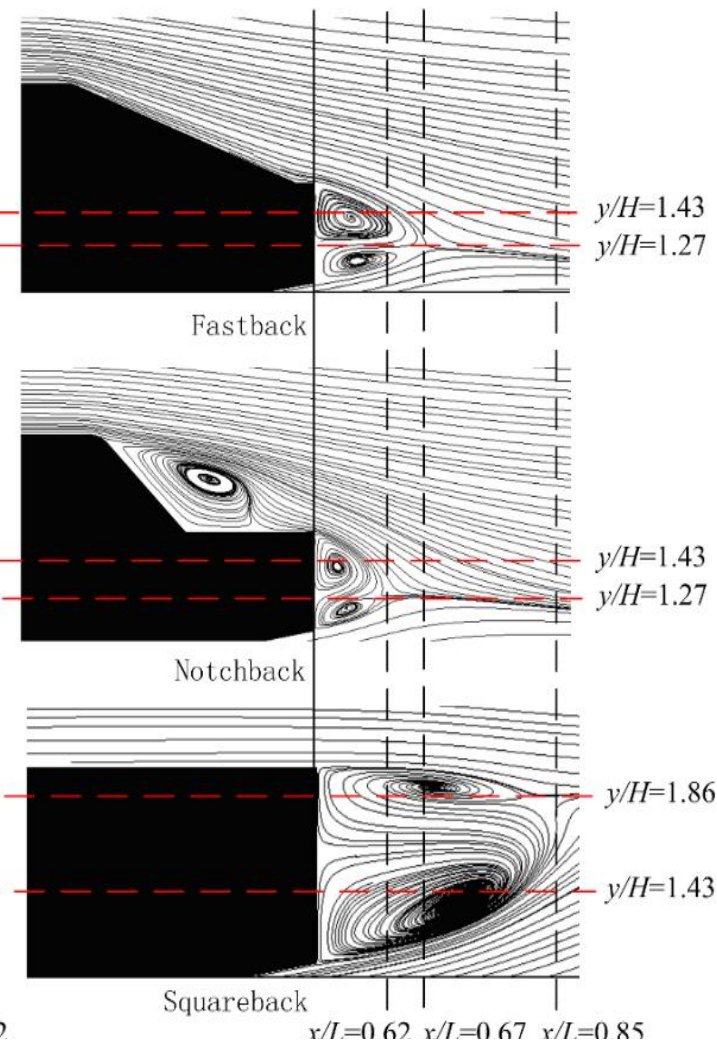

(b) CFD

Fig. 18. The streamline distribution along the longitudinal symmetry plane

Figure 19 shows the turbulent kinetic energy (TKE) which was obtained by CFD on the cross-stream. It is well known that the TKE directly represents the 'strength' of the turbulence in the flow. The contour of the TKE revealed that the turbulent strength diminished gradually with distance, the maximum TKE was located at the vortex core, and at the same position, the turbulent strength of the squareback was the largest, and the fastback was the least. 


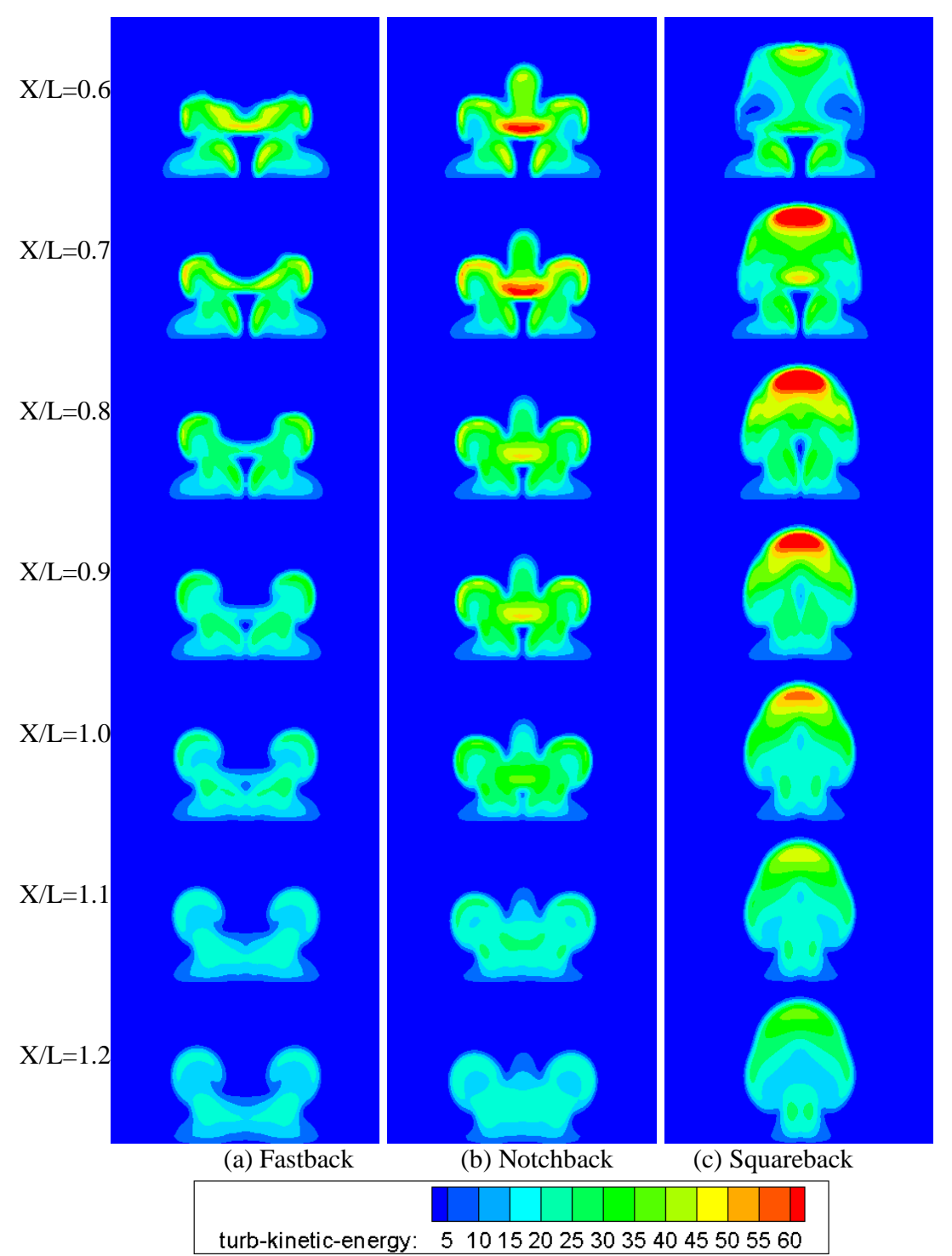

Fig. 19. Turbulent kinetic energy (TKE) on the cross-stream

Figure 20 shows the streamwise velocity profiles at several downstream locations in the symmetry of the near wake of the three models. Note that the model base was located at $\mathrm{x}=694 \mathrm{~mm}$. There was a reversed flow region between $\mathrm{x} \sim 720 \mathrm{~mm}$ and $880 \mathrm{~mm}$ in the wake of fastback and notchback, and between $\mathrm{x} \sim 720 \mathrm{~mm}$ and $1180 \mathrm{~mm}$. The maximum reversed velocities in the recirculation region were approximately 17, 14 and 32 percent of the free stream speed in the wake of the fastback, notchback and squareback respectively.

\section{CONCLUSION}

The numerical simulation and wind tunnel experiment were employed to investigate the aerodynamic characteristic of the three typical passenger cars in current research. In the wind tunnel experiment, the aerodynamic six components were measured with the yaw angle range from $-15^{\circ}$ and $15^{\circ}$, the pressure coefficient on the symmetry plane and wake flow structure with zero yaw angle were also measured by electrical pressure scanning valve and PIV respectively. In the numerical simulation, the realizable $k-\varepsilon$ model with hybrid tetrahedron-Hexahedron-Pentahedral prism mesh strategy to discretize the computational domain was employed to compute the aerodynamic drag, lift and surface pressure distribution within zero yaw angles. The experimental and numerical results revealed that the realizable $k-\varepsilon$ model with hybrid tetrahedron-Hexahedron-Pentahedral prism mesh strategy to discretize the computational domain was proven to be efficient to in simulating the mean flow field around the vehicles. The aerodynamic characteristic is closely related with the rear shape, and different rear shape will induce large different aerodynamic characteristic. The rear negative pressure zone was the main source of aerodynamic drag. Therefore, the drag coefficient of fastback with a more streamlined rear shape was the smallest because of its smaller negative pressure zone. However, the non- streamlined squareback owned 
good stability at high speed and crosswind. The visualization of the wake and the drag coefficient was closely related to both the distance between the model base and the vortex core and the scale of the recirculation zone. The results also revealed that the more non-streamlined the model was, the stronger the turbulence kinetic in its wake.
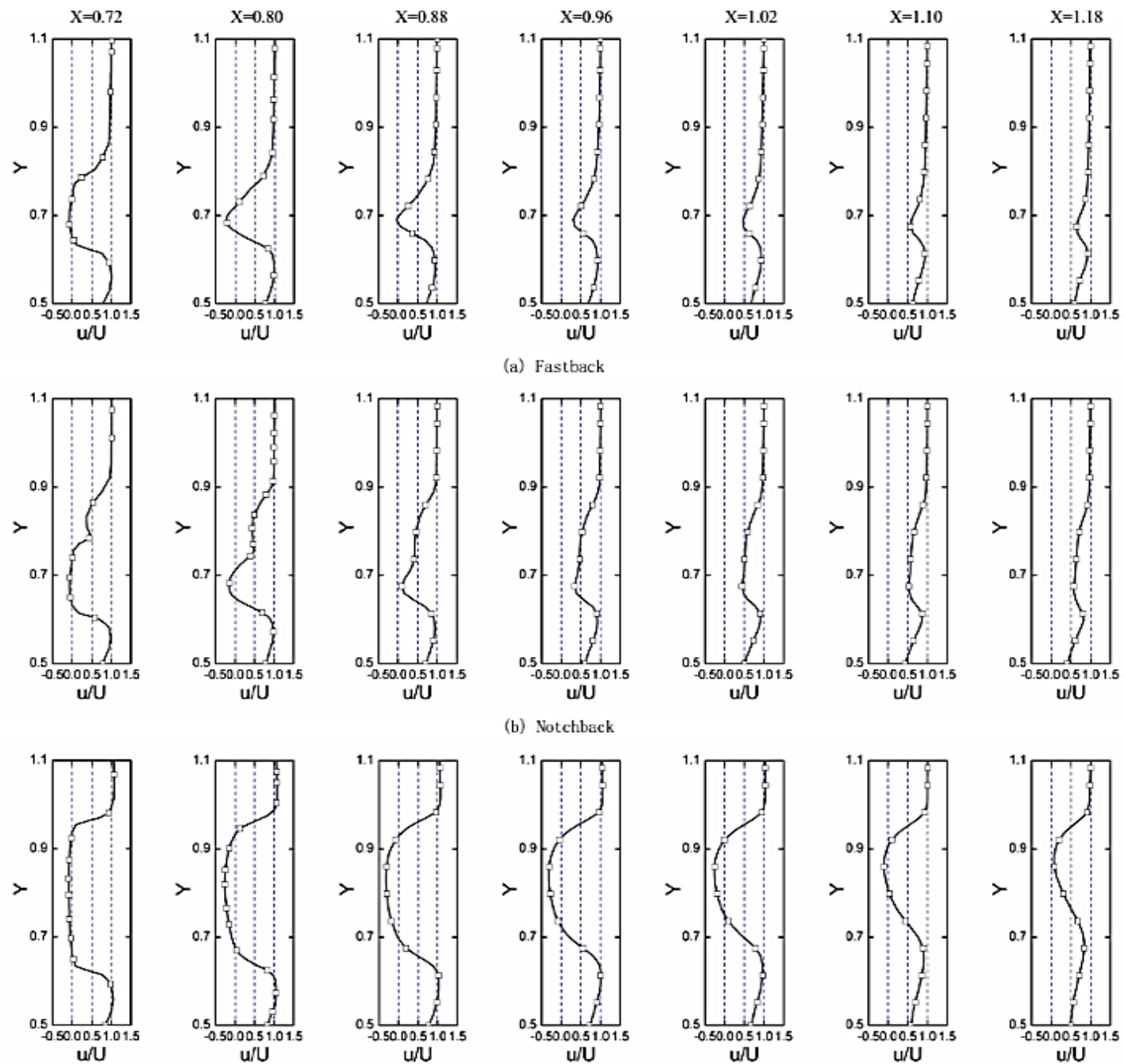

(b) Notchback
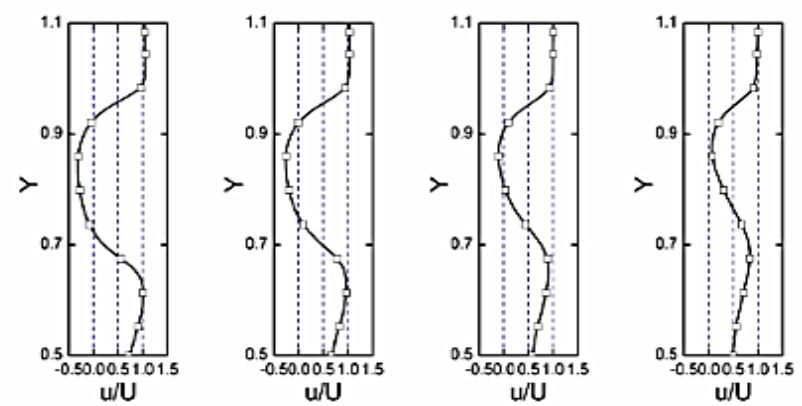

(c) Squareback

Fig. 20. The streamwise velocity profiles at several downstream locations in the symmetry (unit: )m.

\section{ACKNOWLEDGEMENTS}

The research was supported by National Natural Science Foundation of China (Grant No. 51305312), Wuhan Youth Chenguang Program of Science and Technology (Grant No. 2013070104010001), Fundamental Research Funds for the Central Universities (Grant No. 142207005), Hubei Key Laboratory of Advanced Technology of Automotive Components (Grant No. 2012-07) and Foundation of State Key Laboratory of Automotive Simulation and Control (Grant No. 20121111).

\section{REFERENCES}

Abd-Alla, G. H. (2002). Using exhaust gas recirculation in internal combustion engines: a review. Energy Conversion and Management, 43(8), 1027-1042.

Ahmed, S. R. (1983). Influence of base slant on the wake structure and drag of road vehicles. Transactions of ASME, Journal of Fluid Engineering, 105, 429-434.

Ahmed, S. R., G. Ramm and G. Faltin (1984). Some salient features of the time averaged ground vehicle wake. SAE 840300.

Al-Garni A. M. (2008). Measurements of the crossflow velocity field in the wake of an idealized pickup truck model using particle image velocimetry. $14^{\text {th }}$ Int. Symp. on Applications of 
Laser Techniques to Fluid Mechanics, Lisbon, Portugal, 07-10 July, 2008.

Al-Garni, A. M., L. P. Bernal and B. Khalighi (2004). Experimental investigation of the flow around a generic SUV. SAE 2004-01-0228.

Basara, B., Krajnovic S., Girimaji S. and Pavlovic Z. (2012). Near-Wall formulation of the partially averaged Navier-Stokes turbulence model. AIAA Journal, 49 (12), 2627-2636.

Connor, C., A. Kharazi J. Walter and B. Martindale (2006). Comparison of wind tunnel configurations for testing closed-wheel race cars: A CFD Study. SAE 2006-01-3620.

Frederique, M., D. Laurent and H. Vincent (2004). Hybrid method for aerodynamic shape optimization in automotive industry. Computer \& Fluids, 33(5-6), 849-858.

Gilhome, B. R., J.W. Saunders and J. Sheridan (2001). Time averaged and unsteady nearwake analysis of cars. SAE 2001-01-10407.

Gaylard, A., J. Howell and K. Garry (2007). Observation of flow asymmetry over the rear of notchback vehicles. SAE 2007-01-0900.

Himeno, R. and K. Fujitani (1993) Numerical analysis and visualization of flow in automobile aerodynamics development. Journal of Wind Engineering and Industrial Aerodynamics 47, 785-790.

Howell, J. P (1975).Wake prosperities of typical road vehicles. $2^{\text {nd }}$ AIAA Symposium on Aerodynamics of Sports and Competition Automobiles, Los Angeles.

Hucho, W. H (1993).Aerodynamic of road vehicles. Annual Review of Fluid Mechanics 25,485537.

Hucho, W. H (1998) Aerodynamics of Road Vehicles, 4th Ed., Society of Automotive Engineers.

Hoffman, J., B.Martindale and S. Arnette (2001). Effect of test section configuration on aerodynamic drag measurements. SAE 200101-0631.

Jacques, E.V. P. and P. D. Richard (2010). Numerical sensitivity analysis for aerodynamic optimization: A survey of approaches. Computer \& Fluids, 39(3), 373391, 2010.

Janssen, L.J. and W.H. Hucho (1975).Aerodynamische Entwicklung von VW Golf und Scirocco. ATZ, 77: 1-5.

Jenkins, L.N (2000). An experimental investigation of the flow over the rear end of a notchback automobile configuration. SAE 2000-01-0489.

Jindal, S., B. Khalighi and G. Iaccarino (2005). Numerical investigation of road vehicle aerodynamics using the immersed boundary RANS approach. SAE 2005-01-0546.

Khalighi, B., S. Zhang C. Koromilas et al.(2001) Experimental and computational study of unsteady wake flow behind a bluff body with a drag reduction device. SAE 2001-01B-207.

Kim, J. S., J. Sung and S. Kim (2008). PIV Measurements on the change of the threedimensional wake structures by an air spoiler of a road vehicle. Journal of Visualization 11(1), 45-54.

Kourta. A., and Gillieron, P(2009). Impact of the automotive aerodynamic control on the economic issues. Journal of Applied Fluid Mechanics 2(2), 69-75.

Le Good, G., and K. Garry (2004). On the use of reference models in automotive aerodynamics, SAE 2004-01-1308.

Lee, S. J. and J. H. Choi (2000). Ground Effect on Flow around an Elliptic Cylinder in a Turbulent Boundary Layer. Journal of Fluids and Structures 14(5), 697-709.

Marshall, B. and P. Paul, (2000). Mesh Generation, Handbook of Computational Geometry. Elsevier Science.

Murad, N. M., J. Naser F. Alam and S. Watkins (2004). Simulation of vehicle A-pillar aerodynamics using various turbulence models. SAE 2004-01-0231.

McCallen, R., R. Couch J. Hsu et al.(1991) Progress in reducing aerodynamic drag for higher efficiency of heavy duty trucks, (Class 7-8), SAE Paper 1999-01-2238.

Morel, T (1978). The effect of base slant on the flow pattern and drag of three dimensional 
Y. Wang et al. / JAFM, Vol. 7, No. 4, pp. 659-671, 2014.

bodies with blunt ends. Proceedings of Symposium on Aerodynamic Drag Mechanism of Bluff Bodies and Road Vehicles, Plenum Press, NY, p.191-226, 1978.

Pang, J., Li, Z et al. (2002) Correction methods for automotive model tests in TJ-2 wind tunnel. Automotive Engineering 24(5), 371-375.

Hoffman, J., Martindale, B., Arnette, S et al.(2001) Effect of test section configuration on aerodynamic drag measurements. SAE 200101-0631.

Richards, K (2002). Computational modeling of pollution dispersion in the near wake of a vehicle. Doctoral Dissertation, University of Nottingham, 2002.

Song, K. S., S. O. Kang, S. O. Jun et al. (2012) Aerodynamic design optimization of rear body shapes of a sedan for drag reduction. International Journal of Automotive Technology 13(6), 905-914.
Sims-Williams, D., D. Marwood and A. Sprot (2011). Links between Notchback Geometry, Aerodynamic Drag, Flow Asymmetry and Unsteady Wake Structure. SAE Int. J. Passeng. Cars-Mech. Syst. 4(1), 156-165.

Shin, T. H., W. W. Liou A. Shabbir Z. G. Yang and J. Zhu (1995). A new $k-\epsilon$ eddy viscosity model for high Reynolds number turbulent flows. Computers \& Fluids 24(3), 227-238.

Wiedemann, J., and B. Ewaldt (1989). Turbulence manipulation to increase effective Reynolds numbers in vehicle aerodynamics. AIAA Journal 27(6), 763-769.

Wojciak, J., B. Schnepf T. Indinger and N. Adams (2012). Study on the capability of an open source CFD software for unsteady vehicle aerodynamics. SAE Int. J. Commer. Veh. 5(1), 196-207. 